\title{
PRESENCIA DE GENES fimH Y afa EN AISLAMIENTOS URINARIOS DE Escherichia coli PRODUCTORA DE BETALACTAMASAS DE ESPECTRO EXTENDIDO EN LIMA, PERÚ
}

\author{
Jose Matta-Chuquisapon (10), Esther Valencia-Bazalar (1) ${ }^{1, a}$, \\ Luis Marocho-Chahuayo (iD) ${ }^{2, b}$, Edgar Gonzales-Escalante (iD) ${ }^{3,4, a, c}$, \\ Carlos Raúl Sevilla-Andrade (iD ${ }^{3,5, a}$ \\ 1 Universidad Nacional Mayor de San Marcos, Lima, Perú. \\ 2 Instituto Nacional de Salud del Niño, Lima, Perú. \\ 3 Centro de Investigaciones Tecnológicas, Biomédicas y Medioambientales (CITBM), Universidad Nacional Mayor de San \\ Marcos, Lima, Perú. \\ ${ }^{4}$ Laboratorio de Resistencia Bacteriana, Facultad de Farmacia y Bioquímica, Universidad de Buenos Aires, Argentina. \\ ${ }^{5}$ Grupo de Investigación en Resistencia a los antimicrobianos (MICRESIS), Facultad de Medicina, Universidad Nacional \\ Mayor de San Marcos, Lima, Perú. \\ a Tecnólogo médico; ${ }^{b}$ médico cirujano especialista en Enfermedades Infecciosas y Tropicales; ${ }^{\mathrm{c}}$ magíster en Microbiología. \\ El presente estudio forma parte de la tesis de licenciatura de Matta Chuquisapon J. Frecuencia de genes fimH \\ y afa en Escherichia coli productoras de betalactamasas de espectro extendido aisladas de urocultivos, de la \\ Facultad de Medicina de la Universidad Nacional Mayor de San Marcos, presentada en el 2018 en Lima.
}

\section{RESUMEN}

Con el objetivo de determinar la presencia de los genes fimH y afa en aislamientos urinarios de Escherichia coli productoras de betalactamasas de espectro extendido (BLEE), se realizó un estudio descriptivo, con aislamientos del cepario del proyecto TO-06/09 del Instituto Nacional de Salud del Niño en Lima, Perú. Se incluyeron 75 aislamientos urinarios de Escherichia coli. La identificación de genes se realizó por reacción en cadena de la polimerasa. De los 75 aislamientos, 74 (98,7\%) fueron positivos para el gen fimH y 6 $(8,0 \%)$ fueron positivos para el gen $a f a$. Se evidenció la presencia de los factores de virulencia producidos por los genes fimH y afa en aislamientos urinarios de Escherichia coli productoras de BLEE.

Palabras clave: Escherichia coli Uropatógena; Factores de Virulencia; beta-Lactamasas; Perú (fuente: DeCS BIREME).

\section{PRESENCE OF fimH AND $a f a$ GENES IN URINARY ISOLATES OF EXTENDED-SPECTRUM BETA-LACTAMASES PRODUCING Escherichia coli IN LIMA, PERU}

Citar como: Matta-Chuquisapon J, Valencia-Bazalar E, MarochoChahuayo L, Gonzales-Escalante E, Sevilla-Andrade CR. Presencia de genes fimh y afa en aislamientos urinarios de Escherichia coli productora de betalactamasas de espectro extendido en Lima, Perú. Rev Peru Med Exp Salud Publica. 2020;37(2):282-6. doi: https://doi. org/10.17843/rpmesp.2020.372.4829

Correspondencia: Jose Fernando Matta Chuquisapon; Calle José Santos Chocano 199, Ciudad Universitaria, Bellavista, Callao; josematta5511295@gmail.com

Recibido: 23/09/2019 Aprobado: 15/04/2020 En línea: 10/06/2020

\section{ABSTRACT}

Descriptive study conducted in order to determine the presence of the fimH and afa genes in urinary isolates of extended-spectrum beta-lactamases (ESBL) producing Escherichia coli. Isolates from project TO-06/09 of the Instituto Nacional de Salud del Niño in Lima, Peru were used. A total of 75 urinary isolates of Escherichia coli were included. Gene identification was performed by polymerase chain reaction. From the 75 isolates, 74 (98.7\%) were positive for the fimH gene and $6(8.0 \%)$ were positive for the afa gene. Virulence factors produced by the fimH and afa genes were evident in urinary isolates of ESBL producing Escherichia coli.

Keywords: Uropathogenic Escherichia coli; Virulence Factors; beta-Lactamases; Peru (source: MeSH NLM).

\section{INTRODUCCIÓN}

Las infecciones del tracto urinario (ITU) en niños pueden afectar tanto a las vías urinarias altas como a las vías urinarias bajas, lo que causa desde una cistitis hasta una pielonefritis. Durante la infancia, aproximadamente entre $6 \%$ y $8 \%$ de pacientes pediátricos con síntomas urinarios, tienen una ITU ${ }^{(1,2)}$. La frecuencia varía por diversos factores, como la edad y el sexo, y es más común en niñas y en niños no circuncidados. Las ITU en niños con anomalías 
de vías urinarias, como vejiga neurógena o reflujo vesicoureteral, podrían ocasionar un daño renal irreversible ${ }^{(3,4)}$.

La ITU es causada por un grupo de microorganismos conocidos como uropatógenos, que tienen la capacidad de minimizar la respuesta inmune del hospedero e invadir el aparato urinario, siendo la Escherichia coli uropatógena (UPEC, por sus siglas en inglés) la causa del 85\% de los episodios de cistitis aguda en humanos. Este patotipo posee factores de virulencia que le permiten adherirse e invadir los tejidos, además de determinar la capacidad de infección, cronicidad, recurrencia y la posibilidad de diseminarse a otros tejidos ${ }^{(5,6)}$.

Entre los factores de virulencia más frecuentes de la UPEC se encuentran las fimbrias (P y tipo 1); las adhesinas, como fimH, S, M FIC, Dr/afa, Sfa; y los sistemas de captación de hierro (aerobactinas), alfahemolisina y otras enzimas con actividad proteasa ${ }^{(5)}$. La adhesina fimH está presente con una frecuencia superior al $80 \%$ en las cepas de Escherichia coli causantes de ITU. Esta adhesina es la encargada de generar la adherencia de la bacteria al tejido urinario, favoreciendo de esta manera la colonización y posterior invasión al urotelio ${ }^{(6)}$. La adhesina afa tiene una frecuencia que no supera el $40 \%$ en las UPEC, pero es un elemento clave para el desarrollo de infecciones en niños y embarazadas por su capacidad de ocasionar complicaciones ${ }^{(7)}$. Además, es necesario actualizar constantemente los perfiles de susceptibilidad a los antimicrobianos de las UPEC a fin de realizar un tratamiento empírico adecuado para las infecciones del tracto urinario, ya que estos podrían variar según la procedencia, región geográfica o institución ${ }^{(8)}$.

El principal mecanismo de resistencia frente a betalactámicos en enterobacterias es la producción de una betalactamasa de espectro extendido (BLEE). Las BLEE, al tener la capacidad de hidrolizar a la mayoría de los betalactámicos (excepto carbapenémicos y cefamicinas), resultan ser un patrón de multirresistencia, lo que causa un grave problema terapéutico, que explica su asociación con mayor mortalidad, estancia hospitalaria y aumento de costo económico ${ }^{(9)}$. Por ello, el objetivo de este estudio fue determinar la presencia de los genes fimH y afa en aislamientos urinarios de Escherichia coli productoras de BLEE.

\section{EL ESTUDIO}

Se realizó un estudio descriptivo donde se evaluaron aislamientos urinarios de Escherichia coli productoras de BLEE (las bacterias fueron recolectadas entre agosto de 2012 y enero de 2013) del cepario obtenido del proyecto TO-06/09 del Instituto Nacional de Salud del Niño (Detección y caracterización molecular $\beta$-lactamasas de espectro extendido en $E$. coli y K. pneumoniae aisladas en el Instituto Nacional de Salud del Niño). Se recuperaron 75 aislamientos consecutivos no repetidos, obtenidos de muestras de orina provenientes

\section{MENSAJES CLAVE}

Motivación para realizar el estudio: Las adhesinas (como fimH y afa) son responsables de la colonización, invasión y cronicidad de las infecciones por Escherichia coli uropatógena.

Principales hallazgos: En los aislamientos urinarios de Escherichia coli productoras de BLEE, el gen fimH estuvo presente en el 98,7\% y el gen afa, en el 8,0\% de las muestras.

Implicancias: La presencia de fimH y afa en los aislamientos de Escherichia coli productora de BLEE de pacientes pediátricos podría indicar una relación entre las adhesinas y el grupo etario. Se encontró una posible relación entre la no sensibilidad a la amikacina y el gen $a f a$.

de pacientes pediátricos de los servicios de hospitalización y consultorio externo.

La detección molecular se realizó en el laboratorio de Epidemiología Molecular y Genética del Instituto de Medicina Tropical Daniel A. Carrión, de la Universidad Nacional Mayor de San Marcos (UNMSM). Se usó ADN total como molde. Por el método de reacción en cadena de la polimerasa (PCR, por sus siglas en inglés) se amplificó el gen fimH según el protocolo de Tolentino ${ }^{(10)}$. Para el gen afa se estandarizó un protocolo en este estudio tomando en cuenta la concentración de cebadores, ADN Taq polimerasa, la temperatura de hibridación y la concentración de $\mathrm{ADN}$ molde.

Se utilizó el programa estadístico IBM SPSS Statistics for Windows, versión 25 (IBM Corp., Armonk, N.Y., EUA) para reportar frecuencias absolutas y relativas de las variables de interés que fueron obtenidas de la base de datos del cepario.

El protocolo del estudio fue aprobado por la Escuela de Tecnología Médica de la UNMSM. El estudio sigue los lineamientos de las buenas prácticas y de ética en investigación biomédica.

\section{HALLAZGOS}

De los 75 aislamientos de Escherichia coli productoras de BLEE aisladas de urocultivos, 74 (98,7\%) fueron positivos para el gen fimH, y $6(8,0 \%)$ fueron positivos para el gen afa. En el análisis descriptivo de las variables (Tabla 1), la frecuencia de Escherichia coli productoras del gen fimH según procedencia fue $31,1 \%$ hospitalaria y $68,9 \%$ comunitaria. Mientras que para el gen afa la frecuencia fue $16,7 \%$ de procedencia hospitalaria y $83,3 \%$ comunitaria. Del total de aislamientos, 54 (72\%) pertenecían al sexo femenino y la mediana de la edad fue tres años. No se encontró relación entre los genes de virulencia y las variables género, edad y localización. El perfil de susceptibilidad a los antibióticos en los aislamientos de 
Tabla 1. Distribución general de los genes de virulencia de Escherichia coli

\begin{tabular}{|c|c|c|c|c|}
\hline \multirow{2}{*}{ Características } & \multicolumn{2}{|c|}{ gen $a f a$} & \multicolumn{2}{|c|}{ gen $f i m H$} \\
\hline & Sí (\%) & No (\%) & Sí (\%) & No (\%) \\
\hline \multicolumn{5}{|l|}{ Sexo } \\
\hline Masculino & $2(9,5)$ & $90(90,5)$ & $21(100)$ & - \\
\hline Femenino & $4(7,4)$ & $50(92,6)$ & $53(98,2)$ & $1(1,8)$ \\
\hline Edad (años) & - & & & \\
\hline$<1$ & $1(5,0)$ & $19(95,0)$ & $20(100)$ & - \\
\hline 1 & $1(7,7)$ & $12(92,3)$ & $13(100)$ & - \\
\hline 2 & - & $3(100)$ & $3(100)$ & - \\
\hline 3 & $1(16,7)$ & $5(83,3)$ & $5(83,3)$ & $1(16,7)$ \\
\hline 4 & $1(20,0)$ & $4(80,0)$ & $5(100)$ & - \\
\hline 6 & - & $6(100)$ & $6(100)$ & - \\
\hline 7 & - & $7(100)$ & $7(100)$ & - \\
\hline 8 & $2(40,0)$ & $3(60,0)$ & $5(100)$ & - \\
\hline 9 & - & $1(100)$ & $1(100)$ & - \\
\hline 11 & - & $1(100)$ & $1(100)$ & - \\
\hline 12 & - & $2(100)$ & $2(100)$ & - \\
\hline 13 & - & $5(100)$ & $5(100)$ & - \\
\hline 14 & - & $1(100)$ & $1(100)$ & - \\
\hline \multicolumn{5}{|l|}{ Localización } \\
\hline \multicolumn{5}{|l|}{ Comunitaria } \\
\hline Consulta externa & $4(9,1)$ & $40(90,9)$ & $44(100)$ & - \\
\hline Emergencia & $1(12,5)$ & $7(87,5)$ & $7(87,5)$ & $1(12,5)$ \\
\hline \multicolumn{5}{|l|}{ Hospitalaria } \\
\hline $\begin{array}{l}\text { Medicina } \\
\text { interna }\end{array}$ & - & $5(100)$ & $5(100)$ & - \\
\hline $\begin{array}{l}\text { UCI } \\
\text { neonatología }\end{array}$ & - & $4(100)$ & $4(100)$ & - \\
\hline Ortopedia & - & $2(100)$ & $2(100)$ & - \\
\hline Neumología & - & $1(100)$ & $1(100)$ & - \\
\hline UCI & - & $2(100)$ & $2(100)$ & - \\
\hline Neurología & - & $2(100)$ & $2(100)$ & - \\
\hline Nefrología & - & $2(100)$ & $2(100)$ & - \\
\hline Cardiología & $1(50,0)$ & $1(50,0)$ & $2(100)$ & - \\
\hline Cirugía & - & $1(100)$ & $1(100)$ & - \\
\hline Urología & - & $1(100)$ & $1(100)$ & - \\
\hline Ginecología & - & $1(100)$ & $1(100)$ & - \\
\hline
\end{tabular}

UCI: Unidad de Cuidados Intensivos

Escherichia coli productoras de BLEE se muestra en la Figura 2. Asimismo, se evaluó la relación entre la presencia de los genes de virulencia y la no sensibilidad a los antibióticos, y se encontró una asociación entre la no sensibilidad a la amikacina y la presencia del gen afa (Tabla 2).

En la estandarización de la PCR para el gen $a f a$, se realizó una gradiente de temperaturas y se obtuvo que la mejor temperatura de hibridación fue $62{ }^{\circ} \mathrm{C}$, con una concentra-
Tabla 2. Genes de virulencia según susceptibilidad antibiótica

\begin{tabular}{|c|c|c|c|c|}
\hline \multirow{2}{*}{ Antibióticos } & \multicolumn{2}{|c|}{ gen $a f a$} & \multicolumn{2}{|c|}{ gen fimH } \\
\hline & Sí (\%) & No (\%) & Sí (\%) & No (\%) \\
\hline \multicolumn{5}{|l|}{ Amikacina } \\
\hline No sensible & $3(33,3)$ & $6(66,7)^{*}$ & $9(100)$ & - \\
\hline Sensible & $3(4,5)$ & $63(95,5)$ & $65(98,5)$ & $1(1,5)$ \\
\hline \multicolumn{5}{|l|}{ Gentamicina } \\
\hline No sensible & $3(6,7)$ & $42(93,3)$ & $45(100)$ & - \\
\hline Sensible & $3(10,0)$ & $27(90,0)$ & $29(96,7)$ & $1(3,3)$ \\
\hline \multicolumn{5}{|l|}{ Ciprofloxacino } \\
\hline No sensible & $6(8,8)$ & $62(91,2)$ & $67(98,5)$ & $1(1,5)$ \\
\hline Sensible & - & $7(100)$ & $7(100)$ & \\
\hline \multicolumn{5}{|l|}{ Imipenem } \\
\hline No sensible & - & - & - & - \\
\hline Sensible & $6(8,0)$ & $69(92,0)$ & $74(98,7)$ & $1(1,3)$ \\
\hline \multicolumn{5}{|l|}{ Meropenem } \\
\hline No sensible & - & - & - & - \\
\hline Sensible & $6(8,0)$ & $69(92,0)$ & $74(98,7)$ & $1(1,3)$ \\
\hline \multicolumn{5}{|l|}{$\begin{array}{l}\text { Sulfametoxazol- } \\
\text { trimetropima }\end{array}$} \\
\hline No sensible & $5(9,3)$ & $49(90,7)$ & $53(98,5)$ & - \\
\hline Sensible & $1(4,8)$ & $20(95,2)$ & $21(100)$ & - \\
\hline \multicolumn{5}{|l|}{ Nitrofurantoína } \\
\hline No sensible & $1(14,3)$ & $6(85,7)$ & $7(100)$ & - \\
\hline Sensible & $5(7,4)$ & $63(92,6)$ & $67(98,5)$ & $1(1,5)$ \\
\hline
\end{tabular}

${ }^{*}$ Valor de $\mathrm{p}<0,05$ con la prueba de Chi cuadrado.

ción mínima de cebadores de $1 \mu \mathrm{M}$ y de ADN Taq polimerasa de $0,5 \mathrm{U} / \mathrm{rx}$. La concentración mínima de ADN detectable por el test fue $400 \mathrm{pg} / \mathrm{dL}$ (Figura 1).

\section{DISCUSIÓN}

Los resultados del presente estudio muestran que el 98,7\% de los aislamientos de Escherichia coli productoras de BLEE recuperadas de urocultivo de una población pediátrica presentó el gen fimH. Este hallazgo es similar a lo reportado por Kim, et al. ${ }^{(11)}$ al observar la presencia de la adhesina fimH en el total de aislamientos de Escherichia coli en urocultivos de niños, además encontró una relación entre esta adhesina y los filogrupos B2 y D. Asimismo, Tabasi, et al. ${ }^{(12)}$ encontraron, luego de estudiar asilamientos de pacientes adultos con ITU, que $100 \%$ de aislamientos de Escherichia coli portaban del gen fimH a partir de aislamientos de pacientes con ITU en población adulta. De igual modo, Rahdar, et al. ${ }^{(13)}$ detectaron el gen fimH en un $95 \%$ de los aislamientos de UPEC y no hallaron relación entre la presencia del gen fimH y los filogrupos de Escherichia coli. En 2009, Berry, et al. describieron el mecanismo de acción de la adhesina fim $H$, la cual actúa e interacciona con el urotelio, lo que permite el ingreso de la UPEC y la formación de colonias bacterianas 


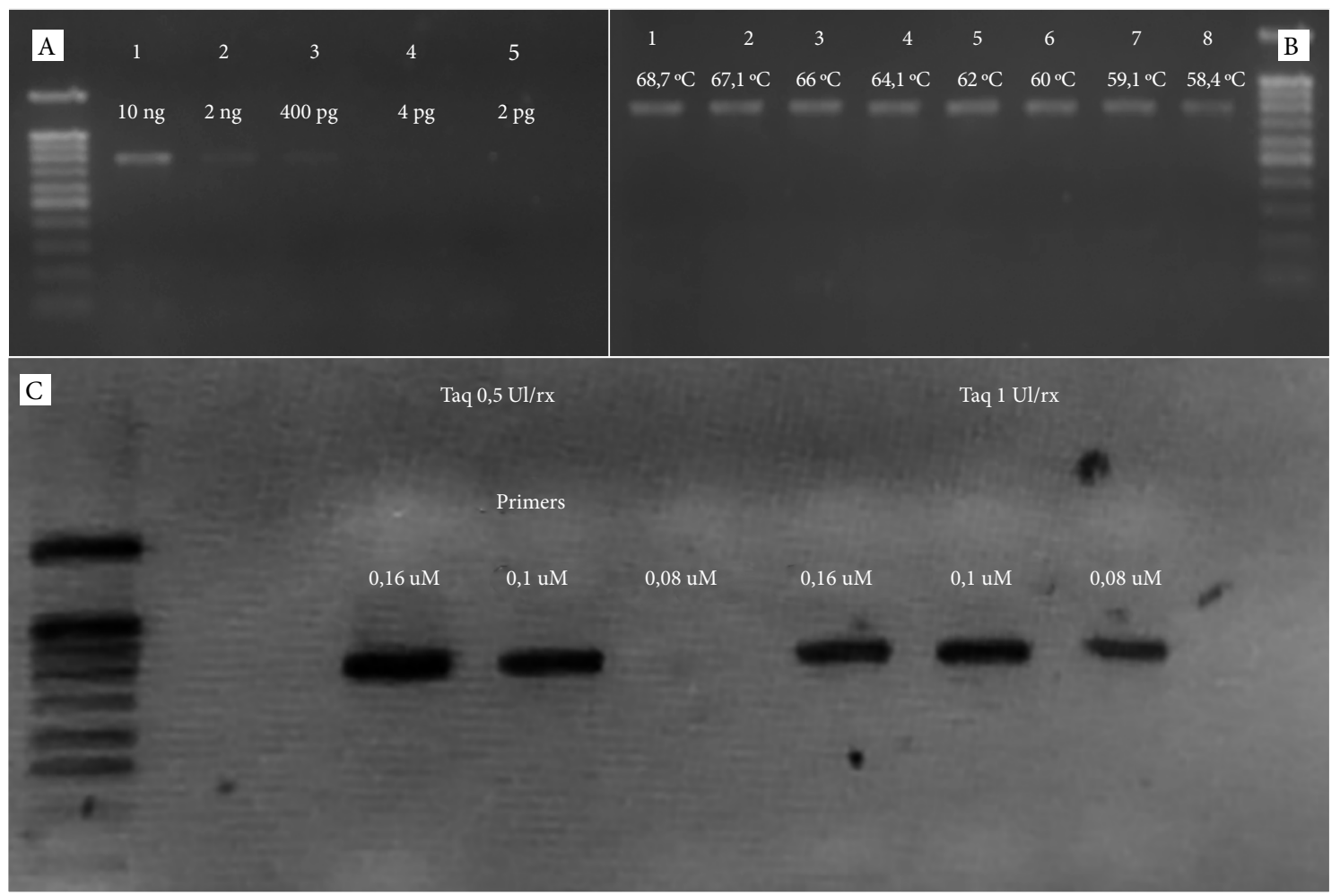

Figura 1. Estandarización del ADN reacción en cadena de la polimerasa para el gen afa en gel agarosa $2 \%$ ladder de $100 \mathrm{pb}$. A. Gradiente de concentración para evaluar la sensibilidad de la PCR para el gen afa (peso molecular del gen afa 750 pb). La concentración mínima detectada fue $400 \mathrm{pg}$. B. Gradiente de temperatura para la estandarización de temperatura de hibridación. C. Estandarización simultánea de la concentración de cebadores y ADN Taq polimerasa. Se concluyó que la concentración óptima de cebadores es $0,1 \mu \mathrm{M}$ con 0,5 UI de concentración de Taq.

intracelulares (CBI) después de las primeras de seis horas de infección ${ }^{(14)}$. Las CBI son responsables de la recurrencia, la cronicidad y la formación de reservorios bacterianos en el urotelio ${ }^{(15)}$

En relación con el gen afa, reportamos una frecuencia de $8,0 \%$ en las UPEC estudiadas; esto concuerda con lo hallado por Ramírez ${ }^{(16)}$, que reportó una frecuencia de $8,2 \%$ del gen afa en cepas UPEC multirresistentes. En contraste, Tabasi, et al. ${ }^{(12)}$, reportan una frecuencia del $29,5 \%$ para el gen $a f a$, y una relación entre cistitis y las infecciones recurrentes con la presencia del gen afa. Por su parte, Servin considera que la presencia del gen afa (subtipo afaE) se presenta en mayor frecuencia en la Escherichia coli que causa pielonefritis ${ }^{(7)}$. Tajbakhsh, et al. reportan que el $32 \%$ de los aislamientos de UPEC tenían el gen afa, además encontró asociación significativa entre la presencia de afa y la producción de biofilms $(\mathrm{p}<0,05)$, característica que además estaba asociada a la presencia de betalactamasas ${ }^{(17)}$.

En 2017 Souza, et al. reportaron una frecuencia del gen afa en aislamientos UPEC de $9 \%{ }^{(18)}$, pero no encontraron una asociación significativa entre el gen y el filogrupo, ni con el género del paciente de donde se aisló. Además, mostraron que la presencia del gen afa y la no sensibilidad a la amika- cina estaban asociadas significativamente, lo que difiere con lo reportado por Malekzadegan, et al. ${ }^{(19)}$, quienes no encontraron asociación entre el gen afa y la no sensibilidad a la amikacina, pero sí entre la presencia del gen afa y la producción de BLEE.

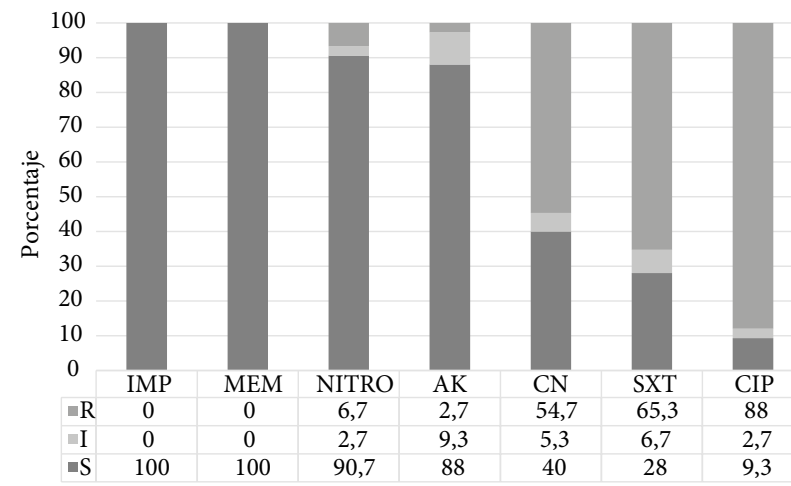

Figura 2. Perfil de resistencia a los antimicrobianos de los aislamientos de Escherichia coli productoras de BLEE $(\mathrm{n}=75)$. R: resistente; I: intermedio; S: susceptible; IMP: imipenem; MEM: meropenem; NITRO: nitrofurantoína; AK: amikacina; $\mathrm{CN}$ : gentamicina; SXT: sulfametoxazol-trimetoprima; CIP: ciprofloxacino. 
El estudio presenta algunas limitaciones que son relevantes mencionar. Los resultados obtenidos corresponden a un solo centro, que podrían diferir según cada población e institución; además, no se buscaron otros factores de virulencia de importancia en la población pediátrica, ni su posible relación con los marcadores de resistencia.

En conclusión, se evidenció la presencia de los factores de virulencia producidos por los genes fimH y afa en aislamientos urinarios de Escherichia coli productoras de BLEE. Además, la estandarización y optimización de la PCR para la detección del gen afa tuvo un buen desempeño.

\section{REFERENCIAS BIBLIOGRÁFICAS}

1. Kaufman J, Temple-Smith M, Sanci L. Urinary tract infections in children: an overview of diagnosis and management. BMJ Paediatr Open. 2019;3(1):e000487. doi: 10.1136/bmjpo-2019-000487.

2. Leung AKC, Wong AHC, Leung AAM, Hon KL. Urinary Tract Infection in Children. Recent Pat Inflamm Allergy Drug Discov. 2019;13(1):2-18. doi: 10.2174/1872213X13666181228154940.

3. Tullus K. Fifteen-minute consultation: ¿Why and how do children get urinary tract infections?. Arch Dis Child Educ Pract Ed. 2019;104(5):2447. doi: 10.1136/archdischild-2018-315023.

4. Asociación Española de Pediatría. Protocolos diagnósticos y terapéuticos en Pediatría: Infectología pediátrica [Internet]. España: ERGON; 2011 [citado el 13 de marzo de 2020]. Disponible en: https://www.aeped. es/documentos/protocolos-infectologia-en-revision.

5. Terlizzi ME, Gribaudo G, Maffei ME. UroPathogenic Escherichia coli (UPEC) Infections: Virulence Factors, Bladder Responses, Antibiotic, and Non-antibiotic Antimicrobial Strategies. Front Microbiol. 2017;8:1566. doi: 10.3389/fmicb.2017.01566.

6. Najafi A, Hasanpour M, Askary A, Aziemzadeh M, Hashemi N. Distribution of pathogenicity island markers and virulence factors in new phylogenetic groups of 57 uropathogenic Escherichia coli isolates. Folia Microbiol (Praha). 2018;63(3):335- 43. doi: 10.1007/s12223-017-0570-3.

7. Servin AL. Pathogenesis of human diffusely adhering Escherichia coli expressing Afa/Dr adhesins (Afa/Dr DAEC): current insights and future challenges. Clin Microbiol Rev. 2014;27(4):823-69. doi: 10.1128/ CMR.00036-14.

8. Warren J, Abrutyn E, Hebel J, Johnson J, Schaeffer A, Stamm W. Guidelines for antimicrobial treatment of uncomplicated acute bacterial cystitis and acute pyelonephritis in women. Infectious Diseases Society of America (IDSA). Clin Infect Dis. 1999;29(4):745-58. doi: 10.1086/520427.

9. Paterson DL, Bonomo RA. Extended-spectrum beta-lactamases: a clinical update. Clin Microbiol. 2005;18(4):657-86. doi: 10.1128/CMR.18.4.657686.2005 .

10. Tolentino E. Detección genotípica de los factores de virulencia: Fimbria tipo 1, Fimbria P y alfa hemolisina en Escherichia coli aisladas de urocultivos [tesis de pregrado]. Lima: Facultad de Medicina, Universidad Nacional Mayor de San Marcos; 2015. Disponible en: http://cybertesis. unmsm.edu.pe/bitstream/handle/cybertesis/7519/Tolentino_le\%20-\%20 Resumen.pdf?sequence $=1$ \&isAllowed $=\mathrm{y}$.
Contribuciones de autoría: Todos los autores han participado en la idea de la investigación, concepción del artículo; recolección de datos y material de estudio; y redacción del artículo. Todos los autores aprobaron la versión final del manuscrito y asumen responsabilidad por su contenido.

Agradecimientos: Al personal del Laboratorio de Epidemiología Molecular y Genética de la Universidad Nacional Mayor de San Marcos por su continuo apoyo.

Fuentes de financiamiento: Autofinanciado.

Conflictos de interés: Los autores no tienen ningún conflicto de interés que declarar.

11. Kim D, Subhadra B, Kang H, Woo K, Kim J, Son Y, et al. Virulence properties of uropathogenic Escherichia coli isolated from children with urinary tract infection in Korea. Genes Genomics. 2018;40(6):625-34. doi: 10.1007/s13258-018-0664-6.

12. Tabasi M. Genotypic Characterization of Virulence Factors in Escherichia coli Isolated from Patients with Acute Cystitis, Pyelonephritis and Asymptomatic Bacteriuria. J Clin Diagn Res. 2016;10(2):1-7. doi: 10.7860/ JCDR/2016/21379.9009.

13. Rahdar M, Rashki A, Miri HR, Rashki Ghalehnoo M. Detection of pap, sfa, afa, foc, and fim Adhesin-Encoding Operons in Uropathogenic Escherichia coli Isolates Collected From Patients With Urinary Tract Infection. Jundishapur J Microbiol. 2015;8(8):e22647. doi: 10.5812/jjm.22647.

14. Berry R, Klummp D, Schaeffer A. Urothelial cultures support Intracellular Bacterial Community Formation by uropathogenic Escherichia coli. Journal Infect Inmun. 2009; 77(7):2762-72. doi: 10.1128/LAI.00323-09.

15. Hanna T, Totsika M, Mansfield K. Host-Pathogen Checkpoints and Population Bottlenecks in Persistent and Intracellular Uropathogenic E. coli Bladder Infection. FEMS Microbiol Rev. 2012;36(3):616-648. doi: 10.1111/j.1574-6976.2012.00339.x.

16. Ramírez-Castillo FY, Moreno-Flores AC, Avelar-González FJ, Márquez-Díaz F, Harel J, Guerrero-Barrera AL. An evaluation of multidrug-resistant Escherichia coli isolates in urinary tract infections from Aguascalientes, Mexico: cross-sectional study. Ann Clin Microbiol Antimicrob. 2018;17(1):34. doi: 10.1186/s12941-018-0286-5.

17. Tajbakhsh E, Ahmadi P, Abedpour-Dehkordi E, Arbab-Soleimani N, Khamesipour F. Biofilm formation, antimicrobial susceptibility, serogroups and virulence genes of uropathogenic $E$. coli isolated from clinical samples in Iran. Antimicrob Resist Infect Control. 2016;5(11):1-8. doi 10.1186/s13756-016-0109-4.

18. de Souza da-Silva AP, de Sousa VS, Martins N, da Silva RC, Bonelli RR, Riley LW, et al. Escherichia coli sequence type 73 as a cause of community acquired urinary tract infection in men and women in Rio de Janeiro, Brazil. Diagn Microbiol Infect Dis. 2017;88(1):69-74. doi: 10.1016/j. diagmicrobio.2017.01.024.

19. Malekzadegan Y, Khashei R, Sedigh Ebrahim-Saraie H, Jahanabadi Z. Distribution of virulence genes and their association with antimicrobial resistance among uropathogenic Escherichia coli isolates from Iranian patients. BMC Infect Dis. 2018;18(1):572. doi: 10.1186/s12879-018-3467-0. 